\title{
LA NAISSANCE DE L'ÉCRITURE CHEZ ROBERT PINGET ET CHEZ SES « ÉCRIVAINS » : LES GÉNÉRATEURS DE TEXTE
}

\author{
Czestaw Grzesiak
}

\begin{abstract}
The article seeks to analyse the role and functioning of text generators in the birth and development of writing in the works of Robert Pinget and his fictional writers. There are two types of generators: the initial generator gives rise to a story, while numerous internal generators develop it. A new writer does not gather any preliminary documents or draw up a plan of what he is going to write about. He lets his pen guide him and in this way he experiences an adventure, arrives at a discovery.

Keywords: Robert Pinget; writing; writer; text generator; Nouveau Roman.

Résumé : L'article a pour but de montrer le rôle et le fonctionnement des générateurs de texte dans la naissance et le développement de l'écriture chez Robert Pinget et chez ses écrivains fictifs. Il y en a deux types : le générateur initial constitue le point de départ et fait naître un récit ; de nombreux générateurs intérieurs le relancent et le développent. Le nouveau romancier ne rassemble donc aucune documentation exhaustive et ne fait jamais le plan de ce qu'il va écrire. Il se laisse conduire par la plume et ainsi va-t-il à l'aventure, à la découverte.
\end{abstract}

Mots clés : Robert Pinget ; écriture ; écrivain ; générateur de texte ; Nouveau Roman.

Parmi les principaux représentants du Nouveau Roman, Robert Pinget (1919-1997) n'est pas celui dont on parle le plus et le plus souvent. Si un mot devait définir ce Genevois, venu en France en 1945 où il partageait sa vie entre son appartement parisien et une vieille ferme en Touraine, ce serait sans doute "discrétion ». Il se tenait à l'écart des débats, des polémiques et des critiques parisiens, vivant et travaillant discrètement, loin des foules et du grand monde. C'est pourquoi il n'a jamais accédé au statut de vedette médiatique. Malgré sa discrétion et sa grande modestie, il est pourtant l'une des figures les plus originales de la littérature française de la seconde moitié du $X X^{\mathrm{e}}$ siècle. Ce qui fait l'originalité de son œuvre romanesque, c'est la présence de nombreux personnagesécrivains. Comment ceux-ci écrivent-ils ? Comment naît et se développe leur écriture? Comment procède Robert Pinget?

Les écrivains appelés traditionnels, comme Honoré de Balzac, Gustave Flaubert, Émile Zola ou Roger Martin du Gard, avant de commencer la rédaction de leur œuvre, réunissaient d'habitude - sur le sujet choisi ainsi que sur les thèmes abordés - une documentation aussi complète que possible et, de plus, ils dressaient un plan plus ou moins détaillé. 
Or, Pinget et ses personnages-écrivains se situent juste à l'opposé de cette pratique. Ils ne rassemblent aucune documentation exhaustive et ne font jamais le plan de ce qu'ils vont écrire. Ils se laissent conduire par la plume qui les mène un peu au hasard. Ainsi vont-ils à l'aventure, à la découverte. Leur devise, c'est la spontanéité et la liberté sur la page.

L'écriture et la fiction chez Pinget et chez ses "écrivains » ont le plus souvent pour préalable le VIDE, le RIEN. Pour commencer un récit (écrit ou oral), l'écrivain ou le conteur a besoin d'un "prétexte » qui déclenchera son écriture ou sa parole. En recourant à la terminologie de Jean Ricardou, nous pouvons donner à ces « prétextes » le nom de générateurs de texte (Ricardou 1973 : 75). Leur répertoire est très riche et varié. On peut y placer un mot, une phrase, une locution, une citation, un objet (par exemple, une fiche, une affiche, un album de photos, un tableau, une image, etc.), un fait divers, un animal ou un être humain. Il faut pourtant préciser qu'à côté du générateur initial, qui constitue le point de départ et qui fait naître un récit, il y en a d'autres, parfois même assez nombreux, qui apparaissent à l'intérieur d'un texte écrit ou oral et qui relancent le récit principal, en le développant et en lui donnant de l'ampleur. En somme, ces différents générateurs deviennent les véritables « moteurs » de l'écriture. ${ }^{1}$ Commençons l'analyse par les générateurs initiaux.

Ce procédé, qui consiste à générer un mini-récit, une histoire ou un conte, apparaît déjà dans le premier livre de Pinget. Là, les conteurs d'histoires trouvent toujours un " propos » qui sert de prétexte pour déclencher et produire une histoire. Blimbraz, par exemple, dit à sa femme : " J'ai vu la Marie passer. Elle avait le chapeau à sa mère. Ça a pas fait long feu » (Pinget $1966: 36$ ). Ce court propos, composé de trois phrases simples, génère tout un conte, intitulé " Le cantonnier », consacré à la descendance royale de Marie (Pinget 1966 : 36-38).

Parfois, il suffit d'évoquer une simple allusion liée à la vie passée d'un personnage, notamment - "Dans sa jeunesse, elle avait eu des démêlés avec un chevrier du bourg » - pour bâtir toute une fable, portant sur la liaison d'une grand-mère avec un gardien de chèvres du village (Pinget 1966 : 58-61).

Dans Mahu ou le matériau, il arrive assez souvent qu'un mot ou une expression génère un long fragment sinon un conte tout entier. Par exemple, le mot « sténo » engendre tout un conte et, en même temps, le charge de tous ses emplois possibles ${ }^{2}$ :

Il paraît que tout le monde cherche une place de sténo, il n'y a que la sténo qui compte vraiment, la sténo c'est la vie, elle ne comprend pas que je n'en fasse pas. On fera tout en sténo. On n'aura plus besoin de l'alphabet qui date des Romains. Tu te rends compte ? Elle [Caroline] achète tout en sténo, elle tend son billet à la marchande qui aime beaucoup mieux ça que de l'entendre bafouiller : «Un kilo de sucre en poudre, deux citrons, des pâtes qui ont cette forme, du vinaigre qui a ce goût, du cirage pas trop jaune » ; elle lit le billet en sténo, elle lui met la marchandise dans un filet en sténo [...] (Pinget 1962 : 90).

Comme nous voyons, Pinget s'amuse à faire des jeux de mots. Ainsi, l'une des séquences de Mahu ou le matériau, intitulée «Retournons à Fantoine », est sans doute bâtie autour des noms des personnages : «Lorpailleur » et « Latirail ». La Lorpailleur, qui veut écrire sur Latirail, arrive un jour à Fantoine. Une devanture de confection « l'attire ». Puis, dans le magasin, elle reconnaît Mahu qui essaie une chemise. Elle lui demande quelle est sa

\footnotetext{
1 On observe une méfiance très forte à l'égard de la conception traditionnelle de la création où l'écrivain (ou le poète) accordait une grande importance à l'inspiration. Celle-ci, aussi bien chez Pinget que chez ses personnages-écrivains, a du mal à s'affirmer comme le « moteur » de l'écriture.

2 Cf. Il en est de même avec l'expression « à peu près » (Pinget 1962 : 113-114).
} 
« taille». L'essentiel, lui répond Mahu, est «que cette chemise m'aille» (Pinget 1962 : 74-75). Or, les mots « l'attire », « taille » et « m'aille » deviennent non seulement des générateurs de fiction, mais aussi, dans leurs différentes combinaisons sonores, ils servent à la création des noms de ces deux écrivains fictifs.

Pinget avoue que, dans ses premiers contes, il avait tendance à se fier au hasard pour produire des effets stylistiques inattendus : « Je piquais avec ma plume dans le dictionnaire le mot destiné à servir de point de départ » (Rambures $1978: 134)^{3}$, dit-il. C'est ainsi qu'il a développé, par exemple, tout un conte, dans son premier livre, à partir des mots « alopécie » et « impétrer » (Pinget $1966: 33-38$ ). Quant aux personnages-écrivains, c'est Lattirail qui suit l'exemple de Pinget. Lorsqu'il n'a pas d'idées, il consulte le dictionnaire Larousse. Son regard s'arrête d'abord sur « les mots qui commencent par < pou > : pouvoir, pousser, etc. ", puis sur le mot clou (Pinget 1962 : 31, 34). Les explications de ces mots lui donnent des tas d'idées pour son roman, intitulé Les chercheurs de clous.

Ceux qui écrivent et qui doivent produire un texte savent parfaitement que le plus difficile est de commencer, d'écrire la première phrase. Pinget éprouve la même difficulté : « le plus difficile, c'est la première phrase », constate-t-il dans un de ses entretiens accordés à J.-L. Rambures (Rambures 1978 : 134). En effet, les premiers mots, qui apparaissent dans l'incipit, sont très importants, car ils mettent en mouvement le livre, l'orientent, le dirigent et parfois même le résument ; en un mot, ils décident de tout le reste ; ils décident de son ton, selon la terminologie pingétienne.

Selon ce principe d'écriture est né le roman le plus volumineux de Pinget : L'Inquisitoire. Il commence par les mots suivants : «Oui ou non répondez ». Cette formule, composée de deux adverbes (l'un indiquant une affirmation et l'autre une négation) et d'une forme verbale (exprimant l'ordre), génère pratiquement tout le roman. Voici ce que Pinget lui-même a dit à propos de sa naissance :

Lorsque j'ai décidé d'écrire L'Inquisitoire, je n'avais rien à dire, je ne ressentais qu'un besoin de m'expliquer très longuement. Je me suis mis au travail et j'ai écrit la phrase. Oui ou non répondez qui s'adressait à moi seul et signifiait Accouchez. Et c'est la réponse à cette question abrupte qui a déclenché le ton et toute la suite (Pinget 1972 : 315).

Il arrive que le texte naisse à partir d'une phrase qui annonce la perte de quelqu'un (la mort ou la fuite d'un être aimé) ou de quelque chose (le manque d'une note).

Pour Le Fiston, Pinget a parié avec une voisine qu'il commencerait son nouveau livre par la phrase : «La fille du cordonnier est morte » (Rambures 1978 : 134). En effet, cette phrase engendre la suite du récit, basé sur la disparition définitive de Marie Chinze et, notamment, sur son enterrement - avec tous les participants, leur comportement durant la cérémonie funèbre et, finalement, leur retour du cimetière (Pinget 1981 : 7-18). Avec l'apparition de Monsieur Levert, qui n'a pas participé à l'enterrement de Marie, le lecteur découvre une autre disparition, sans doute moins tragique que celle de Marie, car le père espère toujours que son fils, qui l'avait quitté il y a dix ans, reviendra peut-être un jour. C'est dans cet espoir qu'il décide de lui écrire une longue lettre. Mais il ne sait pas par quoi commencer. Il recommence donc plusieurs fois sa lettre. Il procède pourtant autrement que Pinget : complètement désespéré, il commence par l'évocation de son état psychique qui se reflète d'ailleurs parfaitement dans son écriture :

3 Cf. Le geste de Pinget rappelle un peu celui des dadaïstes qui, pour désigner leur mouvement poétique, ont également pris le mot «dada » par hasard, en le piquant dans le dictionnaire. 
Mon cher fiston. Je recommence. La figure défaite, les lacets dénoués, le paletot flottant, la tignasse hirsute, l'œil pleurard, la tête vide. Cette prison où je suis. Ça recommence. La main qui t'écrit. Perdu la trace. La trace de la trace du. La tête. L'en-tête. Se précipitent sur le mur, s'écrasent, taches sur le mur, trous. Trous de clous. Mur clouté de trous. S'éloignent, se rapprochent. Ma tête clouée, ces trous dans ma tête, le mur. Je ne voulais pas, je voulais. T'écrire. Comme si la nuit dans sa clémence réussissait à réussir à réunir sous le même toit dans sa clémence indéfinie réussissait à réunir sous le même toit. Torture. Trouée. Cette lettre n'arrivera pas à partir. Je l'aurais postée cette nuit. J'ai posé ma plume, j'allais recommencer (Pinget $1981: 27$ ).

« Il était là ce papier, sur la table, à côté du pot, il n'a pas pu s'envoler » (Pinget 1965 : 7).

$C^{\prime}$ est ainsi que commence le récit de Quelqu'un. Il naît également à partir d'un manque, mais, cette fois-ci, il s'agit d'un bout de papier perdu. L'absence de cette note constitue donc la cellule initiale, le point de départ pour l'exploration minutieuse des lieux divers (chambres, escaliers, jardin, garage, grenier, réfectoire, etc.) et, en même temps, elle donne l'occasion de brosser une sorte de monographie de la pension de famille. On fait ainsi la connaissance de la bonne (Marie), de la cuisinière (Mme Sougneau) et d'autres habitués de la pension (Mme Reber, les Erards, les Cointet, Fonfon), sans oublier son directeur (Gaston).

John Tintouin Porridge, qui figure en tant que personnage-écrivain dans Le Renard et la boussole, cherche, lui aussi, un prétexte pour écrire un nouveau livre. Il le trouve par hasard, au cours d'une promenade en ville. C'est « une affiche invitant pour un prix modique les personnes qui s'y intéressent à visiter la Palestine » :

Ce pays lointain a germé dans ma tête, très lointain, je ne me souvenais plus de lui , il évoquait tout à coup des images bibliques, les plus légendaires, elles ont quelque chose d'inaccessible car liées à l'enfance et au catéchisme elles nous semblent perdues pour toujours, et voilà sur cette affiche la possibilité de les rejoindre, de les vérifier (Pinget 1971 : 33).

Le voyage en Israël sera donc pour John une excellente occasion pour visiter le pays, pour vérifier ses connaissances acquises, pour se débarrasser de tous les clichés qu'on a dans la tête et, enfin, pour écrire. Son nouveau livre prendra ainsi la forme d'un journal de voyage.

Quant aux générateurs intérieurs, qui relancent le récit, ils peuvent être assez nombreux et variés.

Dans le cas de L'Inquisitoire, ce sont tout d'abord les nombreuses questions, posées par l'inspecteur et adressées à l'ex-domestique, qui constituent la principale force motrice du récit. Ces questions provoquent sans cesse de nouvelles réponses et mettent en mouvement le mécanisme de la mémoire. Si celle-ci est incapable d'opérer une véritable reconstitution du passé, l'interrogé recourt à son imagination et invente des renseignements qui pourraient satisfaire l'enquêteur. Ainsi s'opère un glissement continuel du souvenir à l'imaginaire (Pinget 1962a : 117-119, 219-222, 239-244, etc.). Il faut pourtant souligner que l'enquêteur n'est pratiquement jamais satisfait des réponses de son interlocuteur, $c^{\prime}$ est pourquoi il le mobilise et lui lance constamment de nouveaux ordres : " répondez », « décrivez », « détaillez », « répétez », " poursuivez », " précisez », « abrégez », etc. Ces ordres et des questions concrètes (concernant les personnages, leurs occupations, leurs relations interpersonnelles, ainsi que l'espace et les objets) relancent l'interrogatoire et entraînent une grande quantité de renseignements, de descriptions, de digressions, de précisions et de détails apparemment gratuits. Chaque réponse - plus étendue et orien- 
tée sur un fait, un personnage ou une donnée spatiale - constitue un mini-récit. L'ensemble de ces mini-récits forme, à son tour, le roman de Pinget.

Le livre pourtant le plus riche en générateurs intérieurs est, sans doute, Quelqu'un. Son narrateur constate même que, pour relancer et continuer le récit, "il fallait trouver un prétexte » : "Qu'est-ce que j'ai trouvé. La machine à laver ? Non. Les vacances ? Non. Fonfon. Ça me revient, j'ai trouvé Fonfon » (Pinget 1965 : 77). Ce prétexte, qu'il soit être humain ou objet, génère la suite du texte.

D'habitude, l'apparition inattendue d'un nouveau personnage devient le prétexte pour sa présentation :

Un mot aussi de madame Erard, puisqu'elle est tombée dans le jeu de quilles. Elle et son mari sont nos troisièmes ou quatrièmes pensionnaires. Ou cinquièmes ? Ils logent à droite de l'escalier quand on arrive au premier. A droite en face. C'est simple, leur chambre est la première en partant du jardin, au-dessus du réfectoire. Lui est représentant, dans la cinquantaine. Elle est dans la trentaine, c'était sa secrétaire dans une affaire de bas qui a foiré. Ils ont régularisé. Comme son salaire à lui ne suffit pas, elle fait des poupées en chiffons qu'elle vend aux aveugles qui les revendent comme leur fabrication. Elle parle tout le temps de combien de poupées il lui reste à faire avant midi ou avant minuit (Pinget 1965 : 93).

Puis, ce sont les repas, surtout le déjeuner et le dîner, qui permettent au narrateur de progresser et d'enrichir son récit par la présentation de tous les pensionnaires (réunis cette fois-ci à table), de leur comportement, des sujets de conversation et, évidemment, des plats successivement servis (Pinget 1965 : 123-156).

Le soir, les pensionnaires passent leur temps à feuilleter l'album de Gaston : " C'est un album de photos. Les photos de famille. De pension de famille" (Pinget $1965: 198)$. Ce nouveau générateur est extrêmement efficace, car les photos permettent au narrateur de brosser l'histoire de la pension de famille. On commence par les fondateurs : $1^{\text {re }}$ photo : Quelqu'un ; $2^{\mathrm{e}}$ photo : son ami, Gaston ; $3^{\mathrm{e}}$ photo : tous les deux ensemble. Viennent ensuite leurs amis de vacances et, enfin, les pensionnaires (Pinget 1965 : 198-207). De plus, chaque photo est commentée par le narrateur qui essaie de dresser une sorte de portrait de la personne représentée. À titre d'exemple, nous citons le commentaire concernant la photo avec Gaston :

Deuxième photo au-dessous, toujours sur la première page, Gaston au même endroit. Chemise Lacoste foncée, pantalon blanc, espadrilles. Lui il ne fait pas semblant d'être décontracté. Il a l'air presque bête à force de naturel vrai, il nage dans une joie profonde, molle. Il était tellement content de notre projet que toute sa nature pas compliquée dégouline pour ainsi dire. Il tient aussi sa cigarette en l'air mais ne fait pas semblant, il s'arrête de fumer pour la photo. Derrière, les bonnes femmes regardent toujours et le fox-terrier pisse contre une roue de la poussette. Il me semble, je n'en jurerais pas, que de loin, pour ne pas avoir l'air, j'avais crié quelque chose de spirituel aux bonnes femmes mais je ne me souviens plus quoi. Ça me surprendrait de n'avoir rien dit, tellement je me connais (Pinget 1965 : 199-200).

La séance s'achève par quelques photos de Noël et de pique-nique (Pinget 1965 : 229232). Aussi bien le narrateur que les pensionnaires tiennent beaucoup à ces photos, car celles-ci sont toute leur vie ; c'est aussi la seule trace qui reste et qui documente leur séjour terrestre.

Pour retrouver sa fiche perdue, Quelqu'un inspecte tous les endroits possibles, y compris la poubelle. Celle-ci peut être considérée comme un mini-générateur qui déclenche un tas de détails sur son contenu et, en particulier, sur tout ce que les pensionnaires consomment : 
Ensuite j'ai vidé la poubelle, un travail infect, j'ai revu tout ce qu'on avait mangé depuis trois jours, comment est-ce que j'ai pu par cette chaleur, l'odeur me prenait à la gorge. Toutes les épluchures de courgettes et d'aubergines, le pourri des tomates, les nerfs recrachés du bifteck, les dégueulasseries des casseroles, les trognons de salades, les coquilles d'œufs, les restes moisis de ratatouille [...], les effilochures du foutoir mélangées à des grosses boules de poussière déjà toutes grasses du jus des assiettes, des papiers restés collés au fond depuis Dieu sait combien de temps, tout, j'ai tout regardé (Pinget $1965: 214$ ).

Dans Quelqu'un, on observe encore le retour assez fréquent de l'expression « Du nerf $»^{4}$, qui appartient au français familier et qui remplit aussi la fonction de générateur. Elle apparaît toute seule ou accompagnée d'un développement :

- Du nerf. Je me suis mis à ma table, j'ai relu ce que j'ai rédigé hier, j' ai tout biffé et j'ai recommencé (Pinget $1965: 64$ ).

- Du nerf. Je suis sorti de la remise [...] (Pinget 1965 : 112).

- Du nerf. Il faut continuer. Toute ma tête. Continuer la journée sans penser aux autres, aux vieilles, aux douces (Pinget 1965 : 222).

- Du nerf. Après l'apéritif avec Gaston je n'avais pas envie de faire grand-chose (Pinget 1965 :

232).

- Du nerf (Pinget 1965 : 252, 253).

La formule « Du nerf » permet donc le retour au fil conducteur, c'est-à-dire qu'elle invite et encourage le narrateur-écrivain à reprendre et à continuer son récit principal, axé sur la recherche de la note perdue. Il convient de préciser qu'à côté de la formule " Du nerf », qui fait progresser et même accélérer le récit, on en rencontre encore une autre : «Du calme ». En voici quelques exemples :

- J'ai peur, je continue à avoir peur. Du calme, du calme (Pinget $1965: 58$ ).

- Du calme. Je vais trop vite. Respirons (Pinget $1965: 126$ ).

- Du calme. Toute ma tête. Rien ne presse (Pinget $1965: 242$ ).

Cette nouvelle formule, au contraire, apaise le créateur et ralentit le cours de son récit.

En somme, chez l'écrivain pingétien et chez Pinget lui-même, tout "prétexte » (que ce soit un mot, une formule, un geste, un fait, une chose ou un être humain) devient bon pour déclencher d'abord un récit, puis pour le relancer et, en général, pour générer un fragment de texte ou le livre tout entier.

D'autre part, Pinget et ses écrivains fictifs éliminent tous les éléments de ce travail préparatoire qui était propre au romancier réaliste, c'est-à-dire le rassemblement des matériaux, la constitution de « dossiers » thématiques, la méthode des fiches, le plan du livre, etc. Dans leur activité littéraire, rien n'est programmé. Leur écriture naît spontanément et elle est soumise, en grande partie, à l'improvisation. Sa progression type, c'est invention / gommage ou invention / variation.

\section{Bibliographie}

Pinget, Robert (1962), Mahu ou le matériau, Paris : Minuit.

PINGET, Robert (1962 a), L'Inquisitoire, Paris : Minuit.

Pinget, Robert (1965), Quelqu'un, Paris : Minuit.

PINGET, Robert (1966), Entre Fantoine et Agapa, Paris : Minuit.

PINGET, Robert (1971), Le Renard et la boussole, Paris : 1971.

Pinget, Robert (1972), "Pseudo-principes d'esthétique », in : Ricardou, Jean Rossum-Guyon, Francoise Van (éd.), Nouveau Roman: hier, aujourd'hui, t. 2

\footnotetext{
4 En 1990, Pinget utilisera cette expression comme titre pour le premier « carnet » de Monsieur Songe.
} 
Pratiques, Paris : U. G. É., 311-350.

PIngET, Robert (1981), Le Fiston, Montreux (Suisse) : L'Age d'Homme.

RAmbuREs, Jean Louis de (1978), Comment travaillent les écrivains, Paris : Flammarion. Ricardou, Jean (1973), Le Nouveau Roman, Paris : Seuil.

\author{
Czesław Grzesiak \\ Instytut Filologii Romańskiej \\ Wydział Humanistyczny \\ Uniwersytet Marii Curie-Skłodowskiej \\ Plac Marii Curie-Skłodowskiej 4 A \\ 20031 Lublin \\ Pologne \\ czeslaw.grzesiak@umcs.lublin.pl
}

